\title{
ARTICLE Chemical cocktails enable hepatic reprogramming of human urine-derived cells with a single transcription factor
}

\author{
Wei Tang ${ }^{1,2}$, Ren Guo ${ }^{2,3}$, Shi-jun Shen ${ }^{1}$, Yang Zheng ${ }^{2,3,4}$, Yu-ting $\mathrm{Lu}^{3,4,5}$, Meng-meng Jiang ${ }^{2,3,4}$, Xue Cui ${ }^{1,2}$, Ci-zhong Jiang ${ }^{1}$ and \\ Xin $\mathrm{Xie}^{1,2,5,6}$
}

Human liver or hepatocyte transplantation is limited by a severe shortage of donor organs. Direct reprogramming of other adult cells into hepatic cells may offer a solution to this problem. In a previous study, we have generated hepatocyte-like cells from mouse fibroblasts using only one transcription factor (TF) plus a chemical cocktail. Here, we show that human urine-derived epithelial-like cells (hUCs) can also be transdifferentiated into human hepatocyte-like cells (hiHeps) using one TF (Foxa3, Hnf1a, or Hnf4a) plus the same chemical cocktail CRVPTD (C, CHIR99021; R, RepSox; V, VPA; P, Parnate; T, TTNPB; and D, Dznep). These hiHeps express multiple hepatocyte-specific genes and display functions characteristic of mature hepatocytes. With the introduction of the large $\mathrm{T}$ antigen, these hiHeps can be expanded in vitro and can restore liver function in mice with concanavalin-A-induced acute liver failure. Our study provides a strategy to generate functional hepatocyte-like cells from hUCs by using a single TF plus a chemical cocktail.

Keywords: reprogramming; hepatic transdifferentiation; human urine-derived cells; chemical cocktail; regenerative medicine

Acta Pharmacologica Sinica (2019) 40:620-629; https://doi.org/10.1038/s41401-018-0170-z

\section{INTRODUCTION}

The liver is the largest internal organ in humans, and liver failure is a life-threatening illness that is among the most common causes of mortality [1]. Treating patients with hepatic failure or liverbased metabolic disorders is expensive and complex. Whole or auxiliary partial liver transplantation is often the only available treatment option for severe, even if transient, hepatic failure or end-stage liver-based metabolic disorders [2]. Unfortunately, liver transplantation is limited by a severe shortage of suitable organs and the risks associated with major surgery $[1,3]$. Hepatocyte transplantation is a promising alternative to whole-organ transplantation [4], and a large number of studies in rodents have shown that liver cell transplantation can reverse hepatic failure [5-7]. In addition to direct clinical uses, functional human hepatocytes can also be valuable tools for pharmaceutical applications. However, the availability of human hepatocytes is also a bottleneck for clinical applications and research. To generate functional and expandable human hepatocytes in vitro independent of donor organs is of great interest.

Numerous studies have reported that human hepatocytes can be generated from human embryonic stem cells (hESCs) or induced pluripotent stem cells (iPSCs) [8-10]. However, the use of hESC-derived hepatocyte-like cells faces ethical and possible immune rejection problems. Fully functional hepatocytes are also relatively difficult to obtain from iPSCs, as the whole process is burdensome, and many key steps can affect the final stage of hepatocyte formation [11]. Furthermore, the tumorigenic nature of pluripotent stem cells still limits their direct clinical application [12]. Fortunately, direct reprogramming of one somatic cell type into another cell type without passing through the pluripotent state may bypass some of these problems and offers new ways of generating functional hepatocytes [11, 13-16].

Direct reprogramming of human fibroblasts into hepatocytes has been achieved in vitro by lentiviral expression of Foxa3, Hnfla, and Hnf4a [15] or through overexpression of Hnfla, Hnf4a, and Hnf6 along with the hepatic maturation factors ATF5, PROX1, and CEBPA [11]. Synthetic modified mRNAs have also been used to reprogram human fibroblasts into hepatocyte-like cells without genomic modification [17]. These fibroblast-derived human hepatocyte-like cells (hiHeps) resemble primary hepatocytes to a certain extent. A hiHeps-based bioartificial liver system has been demonstrated to restore liver function and prolong survival in a porcine acute liver failure (ALF) model [18]. Although effective in inducing hepatic transdifferentiation, viral vector-carried transcription factors (TFs) are still not favorable in therapeutic applications. Previous studies have demonstrated that certain TFs used in hepatic reprogramming of mouse fibroblasts can be replaced with chemical cocktails $[16,19]$. We have successfully generated hepatocyte-like cells from mouse fibroblasts using the chemical cocktail CRVPTD (C, CHIR99021; R, RepSox; V, VPA; P, Parnate; T, TTNPB; and D, Dznep) in combination with a single TF (Foxa1, Foxa2, or Foxa3).

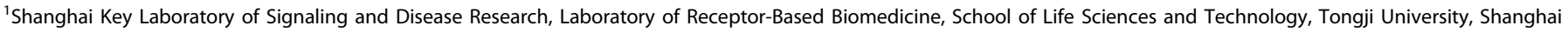

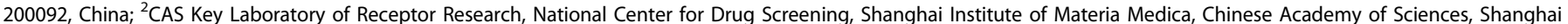

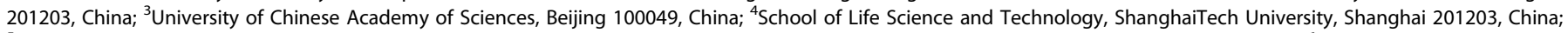

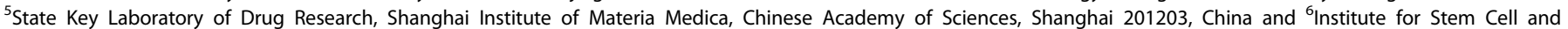
Regeneration, Chinese Academy of Sciences, Beijing 100101, China
} Correspondence: Xin Xie (xxie@simm.ac.cn) 
Here, we report that human urine-derived epithelial-like cells can be directly converted into hiHeps using the same chemical cocktail CRVPTD combined with one TF, namely, Foxa3, Hnf1a, or $H n f 4 a$. These hiHeps are functional both in vitro and in vivo and can rescue mice suffering from ALF.

\section{MATERIALS AND METHODS}

Collection and expansion of human urinary epithelial cells Urine samples were collected from two healthy volunteers (hUC1, a 21-year-old male, and hUC2, a 23-year-old male). The Institutional Review Board approved the collection and use of human samples. Informed consent was obtained from all subjects. Urine was collected with a sterile bottle of proper volume and kept on ice before isolation. The urine sample was centrifuged at $400 \times g$ for $10 \mathrm{~min}$ at room temperature to collect the cells. After careful aspiration of the supernatant, the cell pellet was washed in sterile phosphate-buffered saline (PBS) with 100 units $/ \mathrm{mL}$ penicillin and $100 \mu \mathrm{g} / \mathrm{mL}$ streptomycin and then centrifuged at $400 \times g$ for 10 min. Then, the cell pellet was resuspended in human urine cell medium (hUCM) containing renal epithelial cell growth medium (Lonza, Basel, Switzerland), 10\% fetal bovine serum (FBS; Gibco, Grand Island, NY, USA), 100 units $/ \mathrm{mL}$ penicillin and $100 \mu \mathrm{g} / \mathrm{mL}$ streptomycin. Cells were then transferred into 12-well plates coated with $1 \%$ gelatin solution (Gibco). Approximately $96 \mathrm{~h}$ after plating, most of the medium was aspirated, and $1.5 \mathrm{~mL}$ of hUCM was added. The medium was then changed every other day. The hUCs were collected and split into plates of the appropriate preferred size for further expansion or induction.

\section{Primary hepatocyte culture}

Cryopreserved primary human hepatocytes ( $\mathrm{PHHs}$, product no. M00995-P) were provided by BioreclamationIVT (Baltimore, MD, USA). After thawing, PHHs were cultured in InVitroGRO HI Medium (BioreclamationIVT) with 100 units $/ \mathrm{mL}$ penicillin and $100 \mu \mathrm{g} / \mathrm{mL}$ streptomycin for 2 days before experiments.

Primary mouse hepatocytes were isolated using the classical collagenase perfusion method as described previously [16]. Briefly, the liver was perfused with approximately $25 \mathrm{~mL}$ of perfusion buffer I $\left(0.5 \mathrm{mM}\right.$ EGTA, $16 \mathrm{mM} \mathrm{NaHCO}{ }_{3}$, Hank's balanced salt solution (HBSS) without $\mathrm{Ca}^{2+}$ and $\mathrm{Mg}^{2+}$ ) through the inferior vena and then with approximately $25 \mathrm{~mL}$ of perfusion buffer II $(20 \mathrm{mg}$ collagenase type IV (Gibco), $10 \mathrm{mM}$ HEPES, $16 \mathrm{mM} \mathrm{NaHCO}_{3}, 1 \times$ HBSS with $5 \mathrm{mM} \mathrm{Ca}^{2+}$ and $1.2 \mathrm{mM} \mathrm{Mg}^{2+}$ ). After perfusion, the liver was removed to a $10-\mathrm{cm}$ dish, and hepatocytes were released into Dulbecco's modified Eagle's medium (DMEM) containing 10\% FBS using sterile surgical scissors. The cell suspension was then filtered through a $350-\mu \mathrm{m}$ cell strainer to remove tissue debris. Hepatocytes were purified with Percoll buffer $(5 \mathrm{~mL}$ Percoll (Sigma-Aldrich, St. Louis, MO, USA), $4.5 \mathrm{~mL}$ DMEM and $0.5 \mathrm{~mL}$ $10 \times$ PBS) via low-speed centrifugation $(400 \times g, 15 \mathrm{~min})$; live hepatocytes were collected at the bottom of the tube. The viability of the isolated hepatocytes was approximately $90 \%$, as determined by Trypan blue staining.

\section{Generation of hiHeps}

Plasmids pWPI-Foxa3, pWPI-Hnf1a, pWPI-Hnf4a, and pWPI-large $T$, as well as packing plasmids PSPAX2 and PMD2.0G (gifts from Prof Li-jian Hui, SIBS), were transfected into $293 \mathrm{~T}$ cells. After 48-h incubation, the medium containing the relevant virus was collected and passed through a $0.45-\mu \mathrm{m}$ filter. To generate hiHep cells, urinary epithelial cells were seeded at a density of 400,000 cells per well in a six-well plate and cultured overnight in hUCM. At day 0 , the medium was replaced with virus-containing supernatant supplemented with $4 \mu \mathrm{g} / \mathrm{mL}$ polybrene, and the plates were centrifuged at $400 \times g$ for $90 \mathrm{~min}$ to ensure viral infection. The medium was replaced with fresh hUCM immediately after viral transduction. Two days post viral infection, urine cells were trypsinized into single cells and reseeded at a density of 200,000 cells per well on 12-well plates. At day 3, the medium was replaced with hepatic reprogramming medium (HRM: DMEM/F12, $10 \% \mathrm{FBS}, 10 \% \mathrm{KSR}$ (Gibco), $1 \mu \mathrm{g} / \mathrm{mL}$ insulin, $0.5 \times \mathrm{N} 2$ (Gibco), $0.5 \times$ B27 (Gibco), $2 \mathrm{mM}$ Glutamax, $0.1 \mathrm{mM}$ non-essential amino acids, $100 \mathrm{nM}$ dexamethasone, $10 \mathrm{mM}$ nicotinamide, $20 \mathrm{ng} / \mathrm{mL}$ epidermal growth factor (EGF), $20 \mathrm{ng} / \mathrm{mL}$ hepatocyte growth factor (HGF), 100 units $/ \mathrm{mL}$ penicillin and $100 \mu \mathrm{g} / \mathrm{mL}$ streptomycin) containing the small-molecule cocktail CRVPTD (10 $\mu$ M CHIR99021 (C), $10 \mu \mathrm{M}$ RepSox (R), $0.5 \mathrm{mM}$ VPA (V), $5 \mu \mathrm{M}$ Parnate (P), $1 \mu \mathrm{M}$ TTNPB (T), $50 \mathrm{nM}$ Dznep (D)). The cells were collected for further analysis at day 24. To generate expandable hiHeps, hUCs were infected with virus encoding the large-T antigen in combination with virus encoding Foxa3, Hnfla, or Hnf4a at day 0. Two days after viral infection, hUCs were trypsinized into single cells and reseeded at a density of 50,000 cells per well on 12-well plates. The next day, the medium was replaced with HRM lacking dexamethasone, nicotinamide, EGF, and HGF. At day 18, the cells were reseeded onto new 12-well plates, and the medium was replaced with HRM.

Immunofluorescence staining

Cells were fixed with $4 \%$ paraformaldehyde (PFA) at room temperature for $30 \mathrm{~min}$ and then washed three times with PBS. Then, the cells were blocked with PBS containing $5 \%$ bovine serum albumin and $0.6 \%$ Triton at room temperature for $1 \mathrm{~h}$. Cells were then incubated overnight with the relevant primary antibody at $4{ }^{\circ} \mathrm{C}$. The next day, after thorough washing, the cells were incubated with the appropriate fluorescence-conjugated secondary antibody for $1 \mathrm{~h}$ at room temperature. Nuclei were stained with Hoechst $33342(10 \mu \mathrm{g} / \mathrm{mL})$ for $30 \mathrm{~min}$.

Images were captured on an Olympus IX71 inverted fluorescence microscope. The following antibodies were used in this study: anti-albumin (A80-129A, Bethyl Labs, Montgomery, TX, USA), anti-ZO-1 (33-9100, Thermo Fisher, Waltham, MA, USA), antia-1-antitrypsin (anti-AAT; ab9373, Abcam, Cambridge, MA, USA), anti-Cyp1a2 (AHP610Z, Bio-Rad, Hercules, CA, USA), anti-Cyp2c9 (AHP617Z, Bio-Rad), and anti-Cyp3a4 (AHP622Z, Bio-Rad).

PAS staining, Dil-ac-LDL-uptake assay, and oil red O staining To analyze the glucose storage ability of hiHeps, the cells were fixed with 4\% PFA for $30 \mathrm{~min}$ and stained using the periodic acid-Schiff (PAS) kit (Sigma-Aldrich) according to the manufacturer's instructions. Low-density lipoprotein (LDL)-uptake ability was measured by culturing hUCs and hiHeps in DMEM/F12 containing Dil-Ac-LDL $(10 \mu \mathrm{g} / \mathrm{mL}$, Invitrogen, Carlsbad, CA, USA) for $5 \mathrm{~h}$ at $37^{\circ} \mathrm{C}$. Nuclei were stained with Hoechst, and images were captured on an Olympus IX71 inverted fluorescence microscope. For oil red O staining, cells were fixed in 4\% PFA for $30 \mathrm{~min}$, washed with PBS, stained with oil red O (SigmaAldrich) for $10 \mathrm{~min}$, and then washed with $60 \%$ isopropanol and stained with hematoxylin for $5 \mathrm{~min}$.

\section{Albumin secretion and Cyp450 metabolism assay}

To analyze albumin secretion ability, hUCs, hiHeps, and PHHs were cultured for $24 \mathrm{~h}$, and the supernatants were collected and measured using a human serum albumin kit (Cisbio, Codolet, France) according to the manufacturer's instructions. To measure Cyp450 enzyme activity, hUCs, hiHeps, and PHHs were cultured in media containing $100 \mu \mathrm{M}$ phenacetin and $100 \mu \mathrm{M}$ testosterone for $48 \mathrm{~h}$. Then, the supernatants were collected and mixed with an equal volume of acetonitrile to stop the reaction. After centrifugation at $12,000 \times g$ for $10 \mathrm{~min}$, the concentrations of the metabolites (acetaminophen and $6 \beta$-OH-testosterone) in the supernatants were measured via liquid chromatograph-tandem mass spectrometer (Waters UPLC I-Class and Waters Xevo TQ-S mass spectrometer). All compounds were also purchased from Sigma-Aldrich and used as standard samples. 


\section{Microarray analysis}

Total RNAs extracted from hUCs, PHHs cultured for 2 days, and Foxa3-T-6C-hiHeps at passage 5 from three independent experiments were subjected to whole human gene expression microarray (Agilent SurePrint G3 Human Gene Expression v3 Microarray) according to the manufacturer's instructions. Data were normalized using Gene-Spring version13.1 (Agilent Technologies). Microarray hybridization and analysis were carried out by OUTDO BIOTECH Cooperation (Shanghai, China). Genes with twofold or greater changes $(P<0.05, t$-test) in expression level between PHHs and hUCs (713 genes) were selected to generate the heatmap and for gene ontology (GO) term enrichment analysis.

\section{Cell transplantation in ConA-induced ALF mice}

SCID mice were injected with concanavalin-A (ConA) through the tail vein at a dose of $45 \mathrm{mg} / \mathrm{kg}$ [15]. After $1 \mathrm{~h}$, hiHeps $\left(2 \times 10^{6}\right)$, hUCs $\left(2 \times 10^{6}\right)$, or primary mouse hepatocytes $\left(1 \times 10^{6}\right)$ were injected into ALF mice via the tail vein. Blood and liver samples were collected from the surviving mice at D0, D4, and D7. Alanine transaminase (ALT), aspartate transaminase (AST), and total bilirubin were measured.

\section{Histology}

Fresh liver specimens were fixed overnight with 4\% PFA, after which tissues were embedded in paraffin, cut into $5-\mu \mathrm{m}$ sections, and stored at $4{ }^{\circ} \mathrm{C}$. For hematoxylin and eosin staining, liver sections were dewaxed and rehydrated using a graded ethanol series and then stained with hematoxylin. Afterwards, the sections were dehydrated for eosin staining. Images were captured on an Olympus DP21 microscope.

\section{Real-time PCR}

Total mRNA was isolated using Trizol (Invitrogen), and $1 \mu \mathrm{g}$ RNA was used to synthesize complementary DNA using the PrimeScript RT reagent kit (Takara, Tokyo, Japan) according to the manufacturer's protocol. Real-time PCR was performed using FastStart Universal Probe Master Mix (Roche, Basel, Switzerland) and a StratageneMx 3000P thermal cycler. Primer sequences are supplied in the Supplementary information.

Statistical analysis

Values are reported as mean \pm SEM. Multiple group comparisons were analyzed by analysis of variance, and $P<0.05$ was considered statistically significant. For survival analysis, one-sided Mantel-Cox log-rank tests were applied. All graphs were plotted in GraphPad Prism software.

\section{RESULTS}

Induction of hepatocyte-like cells from human urine cells using single TF with a chemical cocktail

The combination of Foxa3, Hnfla, and Hnf4a has been reported to induce hepatic transdifferentiation of fibroblasts [15]. The removal of any factor from this combination inhibits hepatic transdifferentiation and reduces the expression of hepatic genes. Our previous work has demonstrated that the chemical cocktail CRVPTD (C, CHIR99021; R, RepSox; V, VPA; P, Parnate; T, TTNPB; and $D$, Dznep) can reprogram mouse fibroblasts into iHeps in combination with a single TF [16]. We, therefore, questioned whether this cocktail would also be useful to generate hiHeps with a single TF.

Human urine-derived epithelial-like cells (hUCs) were used as the starting cells since they are easily obtained from urine and efficiently reprogrammed into iPSCs or human neural progenitor cells $[20,21]$. hUC1s (from volunteer 1) and hUC2s (from volunteer 2) both highly expressed the renal epithelial markers CD13 (renal proximal tubule marker), KRT7, SLC2A1, and L1CAM $[22,23]$, indicating that they were renal tubular epithelial cells
(Supplementary Figure S1A). The single factor (Foxa3, Hnf1a, or Hnf4a)-transduced hUC1s were treated with chemical cocktail CRVPTD (6C) for three weeks (Fig. 1a). In addition to 6C, the induction medium HRM was supplemented with $0.5 \times \mathrm{N} 2,0.5 \times$ B27, insulin, dexamethasone, nicotinamide, EGF, and HGF, which have been reported to be beneficial for hepatocyte culture $[16,24]$. Cell morphology changes were observed following induction with one factor and $6 \mathrm{C}$ (Supplementary Figure S1b). In contrast, cells infected with one factor alone did not show significant morphological changes (data not shown). Albumin and a-1-antitrypsin (AAT) were detected in these cells after 6C treatment for 3 weeks (Fig. 1b). Hepatic genes, including Albumin, ASGPR1, CK18, Hnf4a, TTR, and GJB1, were also highly expressed in these hUC1-derived cells at day 24 (Supplementary Figure S1b). These cells resembled induced hepatocyte-like cells reported previously [11, 15], and here, we named them Foxa3-6C-hiHeps, Hnfla-6C-hiHeps, and Hnf4a6C-hiHeps.

PAS staining showed glycogen stores in the Foxa3-6C-, Hnfla6C-, and Hnf4a-6C-hiHeps, and these hiHeps were able to take up LDL (Fig. 1b). Fluorescence-activated cell sorting (FACS) analysis revealed that approximately $27.75 \%, 24.48 \%$, or $22.3 \%$ of Foxa3-, Hnf1a-, or Hnf4a-6C-hiHeps were positive for albumin at day 24, respectively (Fig. 1c). Albumin was also detected in the culture medium of these hiHeps (Fig. 1d). Quantitative RT-PCR analysis showed that the Foxa3-6C-hiHeps, Hnfla-6C-hiHeps, and Hnf4a6C-hiHeps expressed important hepatic Cyp450 enzymes, including Cyp1a2, Сyp3a4, Сyp2b6, Сyp2d6, Сyp2c8, and Сyp2c9, drug transporter genes such as NTCP, MRP2, and the detoxificationrelated nuclear receptors $A H R, P X R, R X R A$, and $R X R B$ (Supplementary Figure S1c).

Hepatic lineage conversion induced by a single factor (Foxa3, Hnfla, or Hnf4a) with $6 \mathrm{C}$ was also confirmed in an additional human urine cell line, hUC2 (from volunteer 2). hUC2-derived hiHeps were able to store glycogen, take up LDL, and secrete albumin (Supplementary Figure S2a and S2b). Hepatic genes, including Cyp450 enzymes, drug transporter genes, and detoxification-related nuclear receptors were also significantly expressed in these hUC2-hiHeps (Supplementary Figure S2C and S2d). Together, these data indicate that $6 \mathrm{C}$ can induce hepatic reprogramming of human urine cells with only one TF.

Generation of proliferative and functional hiHeps by SV40 large T antigen

For further functional characterization and in vivo study, it is important to expand hiHeps in large numbers in vitro. However, the hiHeps derived from hUCs with $6 \mathrm{C}$ and one TF were proliferation arrested (Fig. 1e). Therefore, the SV40 large T antigen (T) was introduced to generate expandable hiHeps, as reported previously [15] (Fig. 2a). hUC1s transduced with a single factor (Foxa3, Hnfla, or Hnf4a), and T grew faster under induction conditions (Supplementary Figure S3a). Foxa3-T-6C-hiHeps, Hnf1aT-6C-hiHeps, and Hnf4a-T-6C-hiHeps were passaged at day 18 and cultured in HRM (Fig. 2a) for further experiments. These T-hiHeps exhibited typical epithelial cell morphology and expressed albumin and AAT (Fig. 2b). Moreover, T-hiHeps acquired mature hepatic functions, including glycogen accumulation, LDL absorption, cytoplasmic accumulation of neutral triglycerides and lipids (Fig. 2b) and albumin secretion (Fig. 2c). RT-PCR analysis showed that the T-hiHeps expressed most hepatic genes, including ASGPR1, Albumin, Transferrin, GJB1, Hnf4a, TTR, ZO-1, Cyp1a2, and Cyp3a4 (Fig. 2d and Supplementary Figure S3b). The renal epithelial markers CD13, KRT7, SLC2A1, and L1CAM were markedly reduced in the T-hiHeps (Supplementary Figure S3c), whereas exogenous Foxa3, Hnfla, and Hnf4a were silenced in the T-hiHeps cultured for 30 days (Supplementary Figure S3d). FACS analysis revealed that approximately $41.13 \%, 25.53 \%$ or $35.29 \%$ of the Foxa3-, Hnf1a-, or Hnf4a-T-6C-hiHeps, respectively, were positive 
a

TF Infection

Foxa3 / Hnf1 / Hnf4a

hUC Culture
Medium: REGM,10\% FBS

D0

b
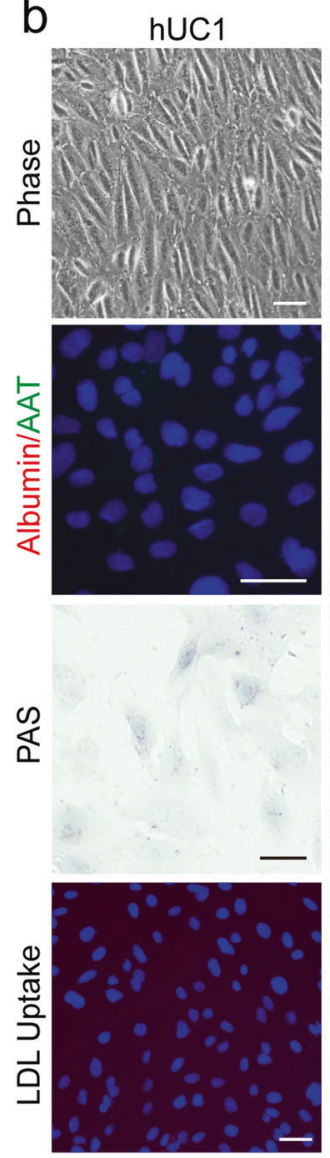

Foxa3+6C
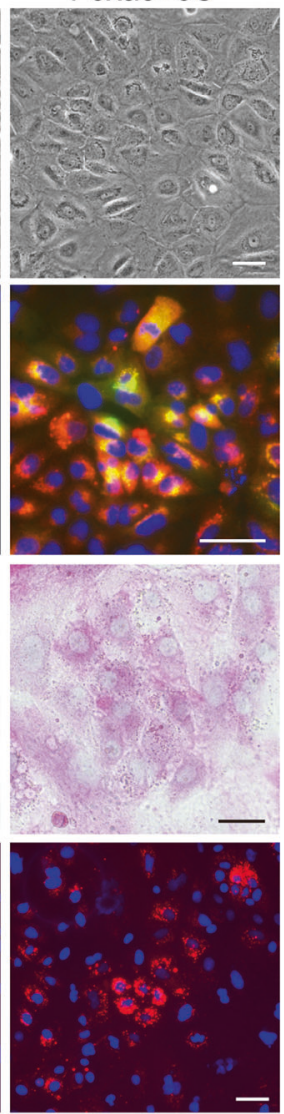

Chemical treatment

(CRVPTD)

HRM: DMEM/F12, FBS, KSR, N2, B27, Insulin, Dex, Nico, EGF, HGF

D3
hiHeps

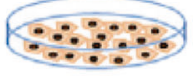

D24

Fig. 1 Hepatic transdifferentiation of hUCs with one TF and chemicals. a Experimental design for the induction of hiHeps. hUCs were infected with lentiviruses expressing the human hepatic transcription factor Foxa3, Hnfla, or Hnf4a. The hUC culture medium was changed to HRM medium containing small molecules (CRVPTD, 6C) 3 days after infection. hiHeps were characterized 24 days after induction. b Representative morphologies of hUC1s (from volunteer 1) infected with Foxa3, Hnf1a, or Hnf4a and treated with $6 \mathrm{C}$ at day 24. Expression of the mature hepatic proteins albumin and AAT were determined by immunofluorescence staining. PAS staining and Dil-ac-LDL (red fluorescence) uptake assays were also performed. Nuclei were stained with Hoechst. c FACS analyses of albumin-positive cells in hUC1s, Foxa3-6C-, Hnf1a-6C-, and Hnf4a-6C-hiHeps at day 24. Data are mean \pm SEM of three independent experiments. ${ }^{* * *} P<0.001$ versus hUC1s. d Analysis of secreted albumin in the culture media of hUC1s, PHHs, Foxa3-6C-, Hnf1a-6C-, and Hnf4a-6C-hiHeps at day 24. Data are mean \pm SEM of three independent experiments. ${ }^{* * *} P<0.001$ versus hUC1s. e Growth curves of cells induced by Foxa3-6C, Hnf1a-6C, or Hnf4a-6C. Data are mean \pm SEM of a representative experiment $(n=3)$ from three independent experiments. Scale bars represent $50 \mu m$. See also Supplementary Figure S1 and S2

for albumin at passage 2 (Fig. 2e). As negative controls, hUCs transduced with large $\mathrm{T}$ plus a single factor (Foxa3, Hnf1a, or $\mathrm{Hnf4a}$ ), or large $\mathrm{T}$ plus $6 \mathrm{C}$ produced no albumin-expressing cells (data not shown).

T-hiHeps were further characterized at passage 5. Cyp450 enzymes, such as Cyp1a2, Cyp3a4, and Cyp2c9, were confirmed by immunofluorescence staining in Foxa3-, Hnfla-, and Hnf4a-T-6ChiHeps (Fig. 3a). Quantitative RT-PCR analysis also revealed that a number of important phase II enzymes, such as UGT1A1, UGT2B15, UGT2B7, phase III transporters, such as NTCP, MRP2, MRP3, and detoxification-related nuclear receptors, such as $A H R, C A R, P X R$, and $R X R G$, were significantly expressed in these T-hiHeps (Fig. $3 \mathrm{~b}$ ). Importantly, the metabolic products of testosterone $(6 \beta-\mathrm{OH}-$ testosterone) and phenacetin (acetaminophen) were detected in the culture media of Foxa3-, Hnfla-, and Hnf4a-T-6C-hiHeps
(Fig. 3c), indicating the acquisition of specific drug metabolism enzymes or pathways. These results show that the T-hiHeps induced by a single TF and $6 \mathrm{C}$ possess typical functional features of hepatocytes.

The Foxa3-, Hnfla-, and Hnf4a-T-6C-hiHeps could be stably expanded in vitro and displayed typical S-shaped growth curves (Supplementary Figure S4a). The mean population doubling time of these T-hiHeps was approximately $24 \mathrm{~h}$, and this growth speed could be maintained for at least 20 passages. In contrast, the growth of hUCs was significantly restricted at passage 5 (Supplementary Figure S4a). At passage 20, these T-hiHeps still expressed albumin and exhibited the ability to store glycogen and absorb LDL (Supplementary Figure S4b and S4c). PCR analysis showed hepatic genes were also highly expressed in these ThiHeps (Supplementary Figure S4d). 
a

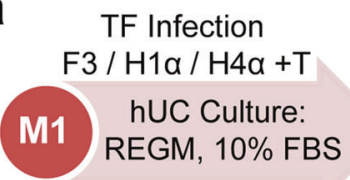

D0
Chemical treatment (CRVPTD)

DMEM/F12, FBS, KSR, N2, B27, Insulin

D3
Expansion

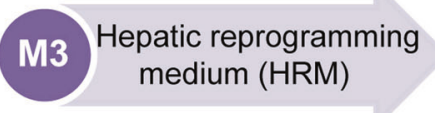

D18 $\mathrm{b}$
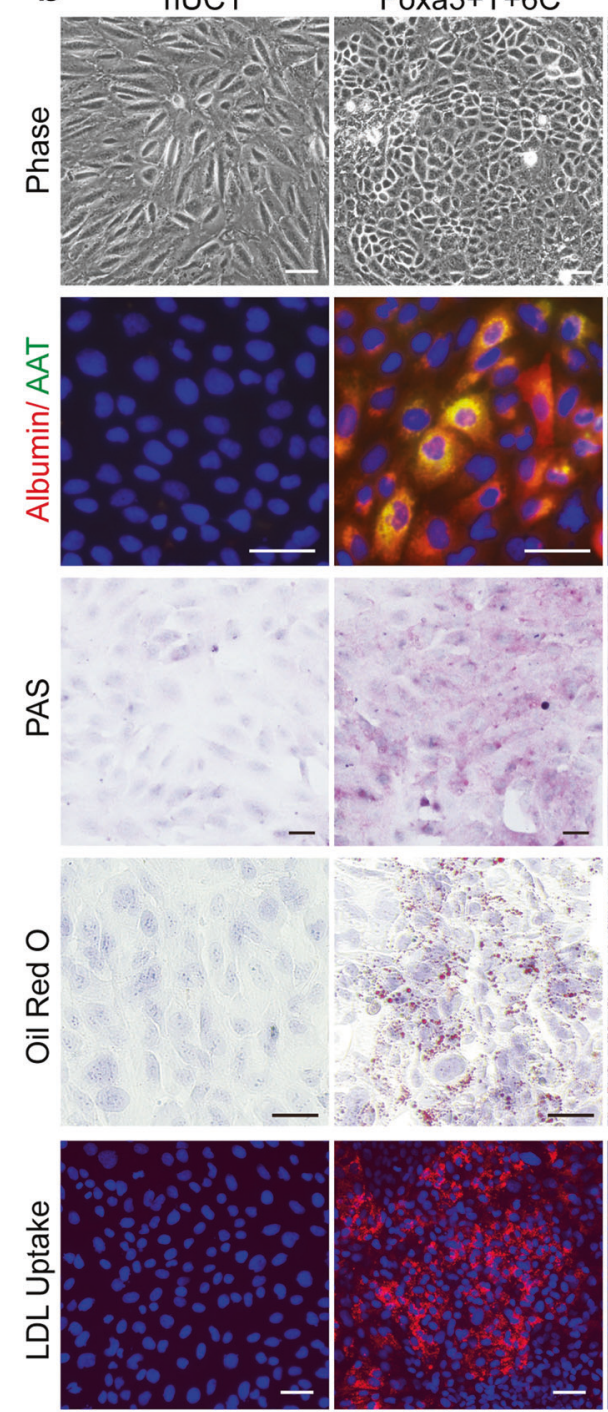

Foxa3 $+T+6 \mathrm{C}$
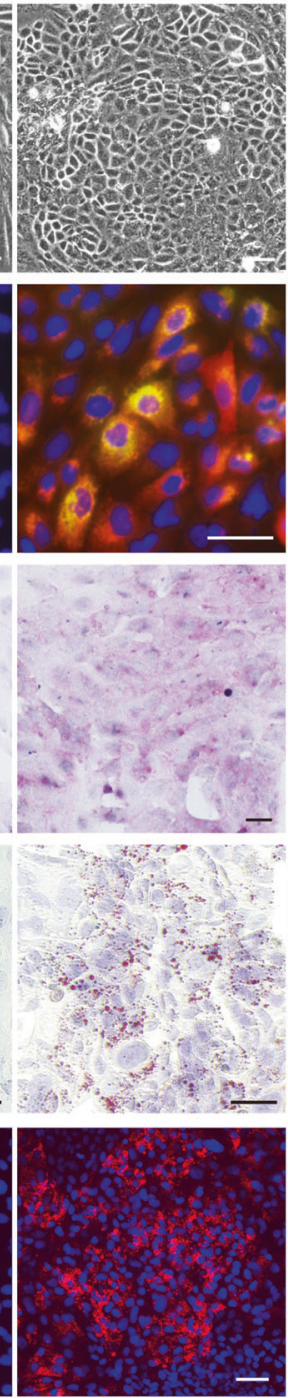

$H n f 1 \alpha+T+6 C$
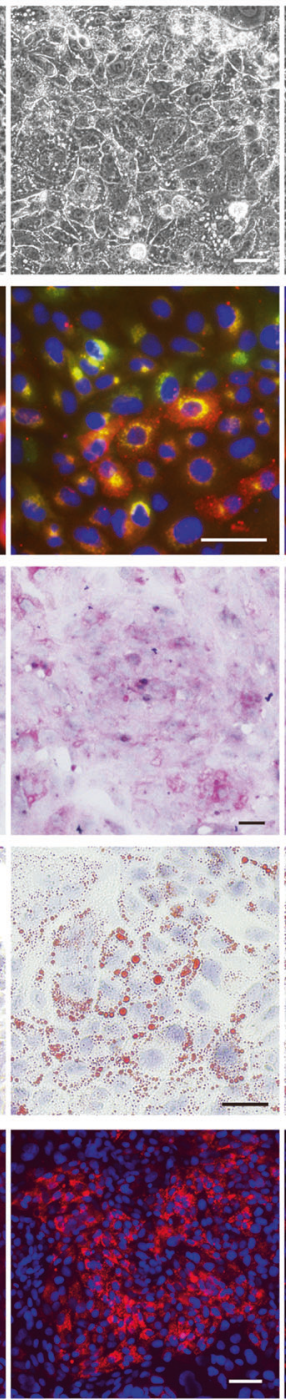

$\mathrm{Hnf} 4 \alpha+T+6 \mathrm{C}$
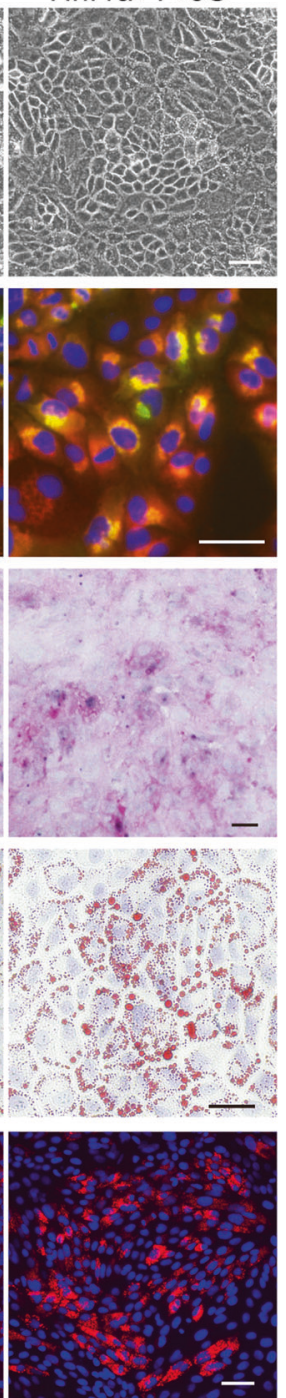

C

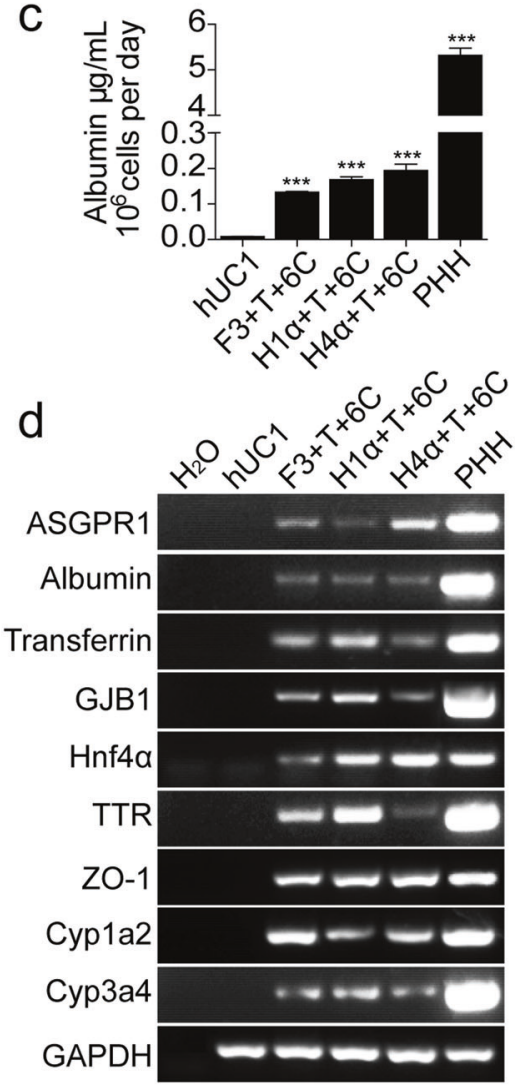

e

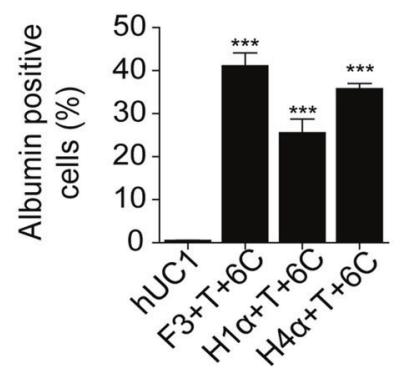

Fig. 2 Generation of expandable hiHeps from hUCs. a Experimental design for the generation of expandable hiHeps from hUCs. hUCs were infected with large-T antigen (T) in combination with Foxa3, Hnfla, or Hnf4a. Small molecules (CRVPTD, 6C) were added 3 days after infection. At day 18, the cells were reseeded for expansion, and the medium was changed into HRM. b Representative morphology of Foxa3-T-6C-, Hnf1a-T-6C-, or Hnf4a-T-6C-hiHeps induced from hUC1s. Albumin and AAT were determined by immunofluorescence staining. PAS staining, oil red $\mathrm{O}$ staining, and Dil-ac-LDL uptake assays were also performed. Nuclei were stained with Hoechst. c Analysis of secreted albumin in the culture media of hUC1s, PHHs, Foxa3-T-6C-, Hnf1a-T-6C-, or Hnf4a-T-6C-hiHeps. Data are mean \pm SEM of three independent experiments. ***P $<0.001$ versus hUC1s. d PCR analysis of hepatic genes in hUC1s, PHHs, Foxa3-T-6C-, Hnf1a-T-6C-, and Hnf4a-T-6C-hiHeps. Data are representative of three independent experiments. e FACS analyses of albumin-positive cells in hUC1s, Foxa3-T-6C-, Hnf1a-T-6C-, and Hnf4a-T$6 \mathrm{C}$-hiHeps at passage 2. Data are mean \pm SEM of three independent experiments. ${ }^{* * *} P<0.001$ versus hUC1s. Scale bars represent $50 \mu \mathrm{m}$. See also Supplementary Figure S3 and S4

Foxa3-T-6C-hiHeps are stably reprogrammed and do not pass an iPSC stage

Genome-wide expression profiling revealed that Foxa3-T-6ChiHeps clustered more closely with cultured PHHs (Fig. 4a). Compared with hUCs, Foxa3-T-6C-hiHeps had 367 genes that were upregulated (Group 1) and 346 genes that were downregulated (Group 2). A GO term enrichment analysis showed that genes involved in metabolic pathways, including oxidation-reduction process, small-molecule metabolism, vitamin metabolism, aminoacid metabolism and fatty acid metabolism, and defense response to virus, were significantly upregulated in Foxa3-T-6C-hiHeps, whereas genes involved in extracellular matrix organization, cell adhesion, regulation of actin cytoskeleton, regulation of epithelial cell migration, and kidney development were significantly 
a
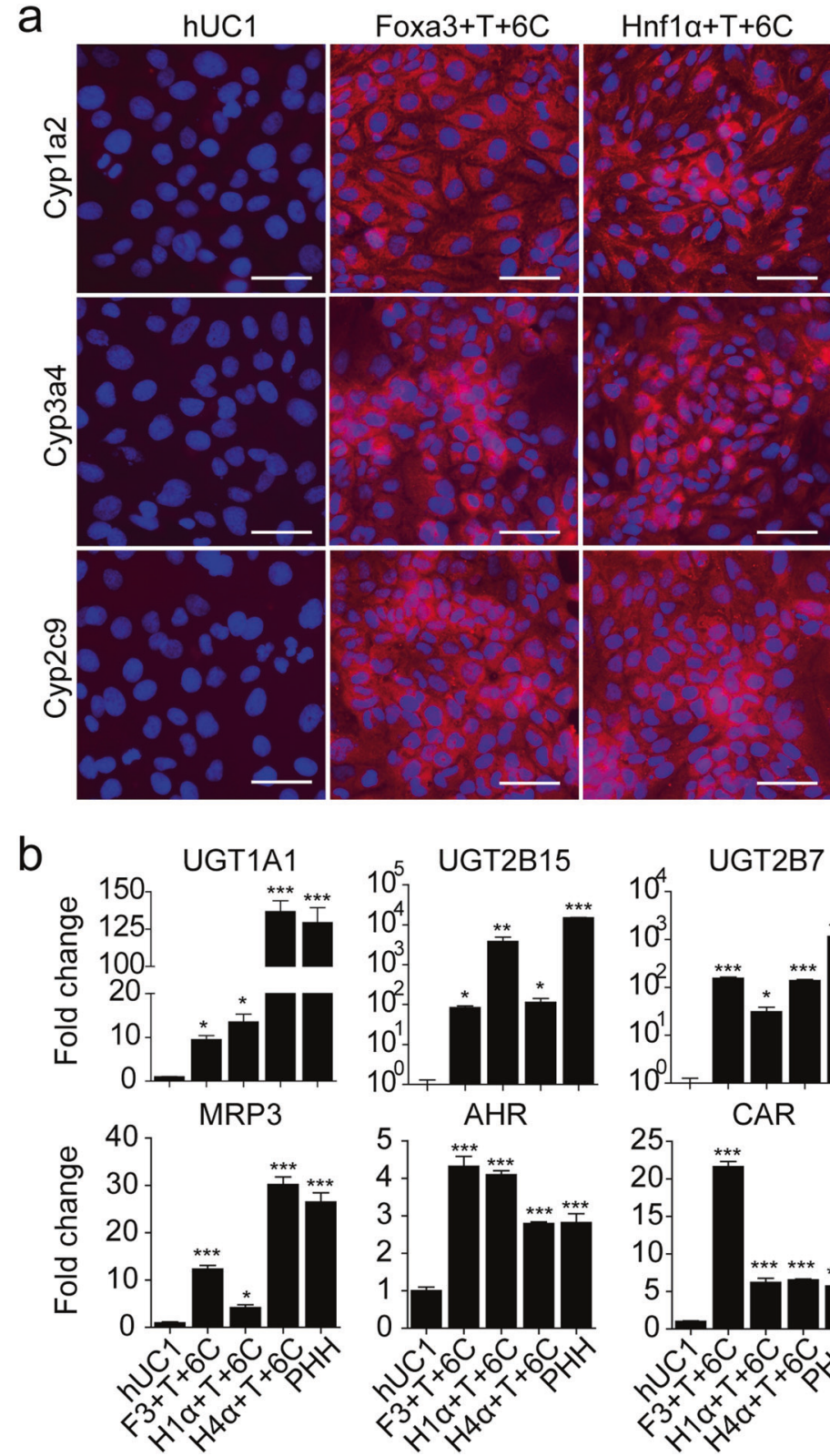
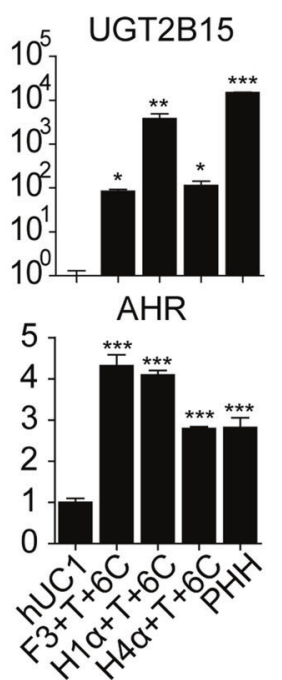

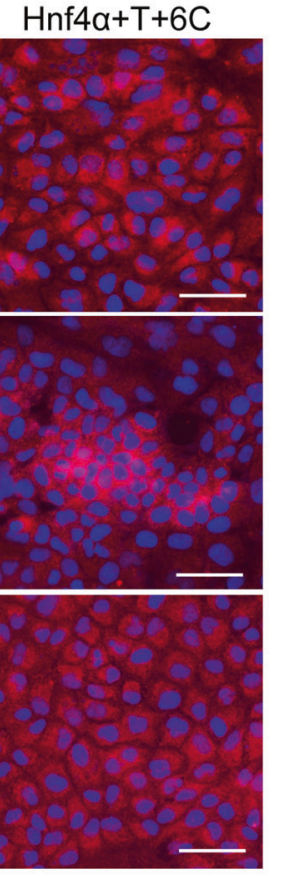

C
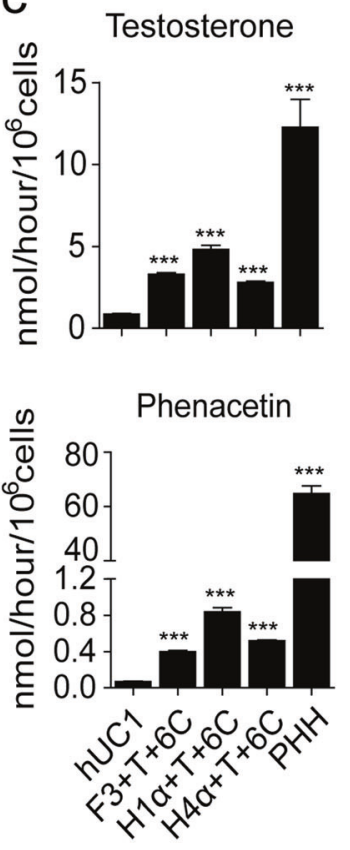

Fig. 3 T-hiHeps possess CYP enzyme activities. a Immunofluorescence staining of hepatic Cyp450 enzymes Cyp1a2, Cyp3a4, and Cyp2c9 in hUC1s, Foxa3-T-6C-, Hnf1a-T-6C-, and Hnf4a-T-6C-hiHeps at passage 5. Nuclei were stained with Hoechst. b Quantitative RT-PCR analysis of the expression of drug metabolic phase II enzymes (UGT1A1, UGT2B15, UGT2B7), phase III transporters (NTCP, MRP2, and MRP3) and detoxificationrelated nuclear receptors ( $A H R, C A R, P X R$, and $R X R G)$ in hUC1s, PHHs, Foxa3-T-6C-, Hnf1a-T-6C-, and Hnf4a-T-6C-hiHeps. Data are mean \pm SEM of

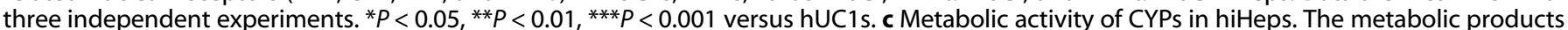
of testosterone (6 $\beta-\mathrm{OH}$-testosterone, assay for Cyp3a activities) and phenacetin (acetaminophen, assay for Cyp1a2 activities) were measured

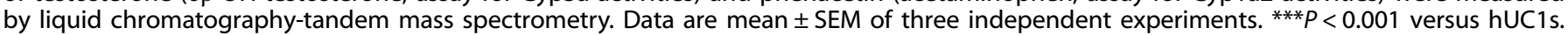
Scale bars represent $50 \mu \mathrm{m}$

downregulated (Fig. 4b), indicating a clear transition from hUCs to a differentiated hepatocyte-like state.

Quantitative RT-PCR analysis revealed the very low expression of endodermal progenitor markers, including GATA4, SOX17, FOXA2, AFP, and hepatoblast markers such as Sox9 and TBX3 during Foxa3-T-6C-hiHeps induction (Fig. 4c). Importantly, the pluripotency genes Nanog, Sox2, and Oct4, which were highly expressed in hESC-H9, also remained at very low levels during Foxa3-T-6C-hiHeps induction (Fig. 4d), indicating that the hepatic transdifferentiation does not pass an iPSC stage. Moreover, the Foxa3-T-6C-hiHeps maintained a normal chromosome karyotype at late passages (Fig. 4e).
Foxa3-T-6C-hiHeps attenuate ConA-induced ALF

$A L F$ is a devastating syndrome with severe liver injury and rapid loss of hepatic function [25, 26]. ALF is associated with significant mortality worldwide $(40 \%-80 \%)$, and $>50 \%$ of patients require liver transplants [27]. The lectin ConA is known to induce fulminant liver injury in mice, which simulates many symptoms of human ALF [27]. ConA is widely used to evaluate the functions and therapeutic effects of hepatocytes, mesenchymal stem cells or hepatic-like cells in ALF [28].

To study the in vivo functions of Foxa3-T-6C-hiHeps, SCID mice were injected with ConA to induce ALF. One hour after induction, hUCs, Foxa3-T-6C-hiHeps, or primary mouse hepatocytes were 


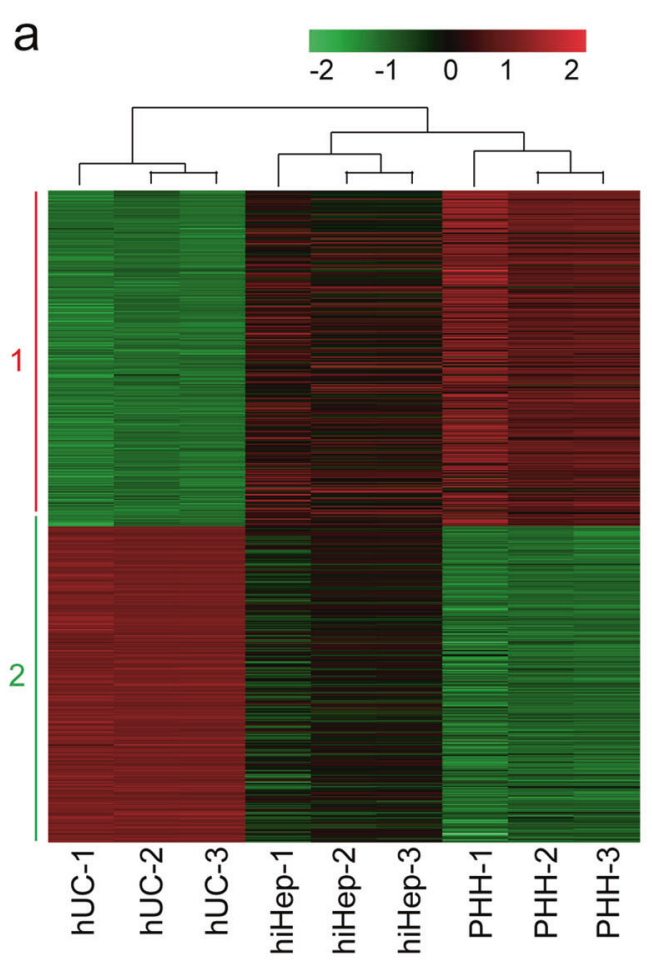

C
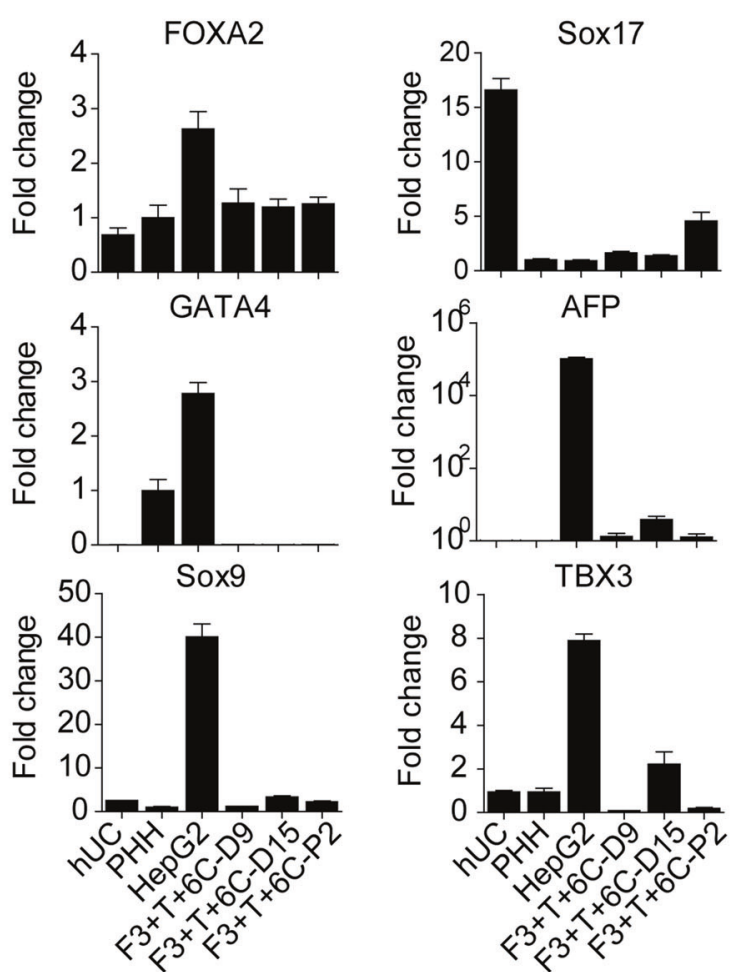

b

\begin{tabular}{|l|}
\hline Oxidation-reduction process \\
\hline Type I interferon signaling pathway \\
\hline Small molecule metabolic process \\
\hline Cytokine-mediated signaling pathway \\
\hline Negative regulation of viral gennome replication \\
\hline Interferon-gamma-mediated signaling pathway \\
\hline Defense response to virus \\
\hline Regulation of complement activation \\
\hline Response to interferon-gamma \\
\hline Response to interferon-beta \\
\hline Water-soluble vitamin metabolic process \\
\hline Vitamin metabolic process \\
\hline Positive regulation of T cell mediated cytotoxicity \\
\hline Fatty acid metabolism \\
\hline Positive regulation of cholesterol esterification \\
\hline High-density lipoprotein particle remodeling \\
\hline Response to virus \\
\hline Response to interferon-alpha \\
\hline Glysine, serine and threonine metabolism \\
\hline Fatty acid degradation \\
\hline
\end{tabular}

upregulated genes

d

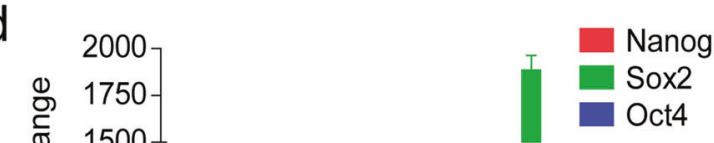

Fig. 4 Genome-wide analysis of Foxa3-T-6C-hiHeps. a Heatmap of microarray data from hUCs, PHHs, and Foxa3-T-6C-hiHeps (at passage 5). Groups 1 and 2 contain genes with > 2-fold upregulation or downregulation in PHHs compared with hUCs. b GO term enrichment analysis of genes that display significant change in expression in Foxa3-T-6C-hiHeps and PHHs compared with hUCs. c Quantitative RT-PCR analysis of the expression of endodermal progenitor markers (GATA4, Sox17, FOXA2), hepatic progenitor gene (AFP) and hepatoblast markers (Sox9 and TBX3) in hUCs, PHHs, HepG2s, and Foxa3-T-6C-hiHeps during induction. Data are mean \pm SEM from a representative experiment $(n=3)$ of three independent experiments. Data are normalized to PHHs. d Quantitative RT-PCR analysis of the expression of human pluripotency markers (Nanog, Sox2, and Oct4) in hUCs, hESC-H9, and Foxa3-T-6C-hiHeps during induction. Data are mean \pm SEM from a representative experiment $(n=3)$ of three independent experiments. Data are normalized to hUCs. e The karyotype of Foxa3-T-6C-hiHeps at passage 10 was analyzed by chromosome analysis during mitosis 
a

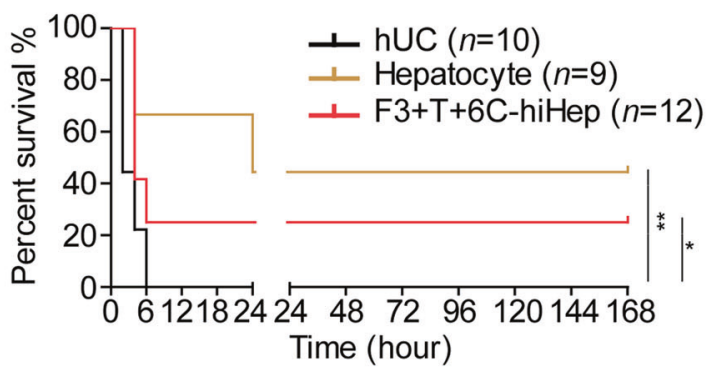

b

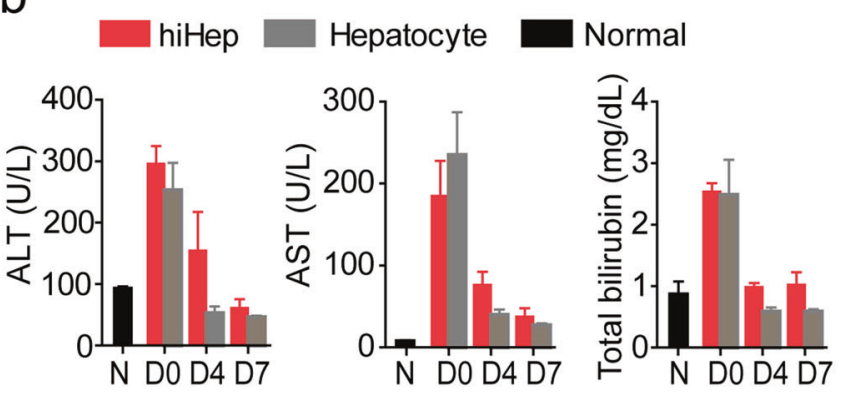

C

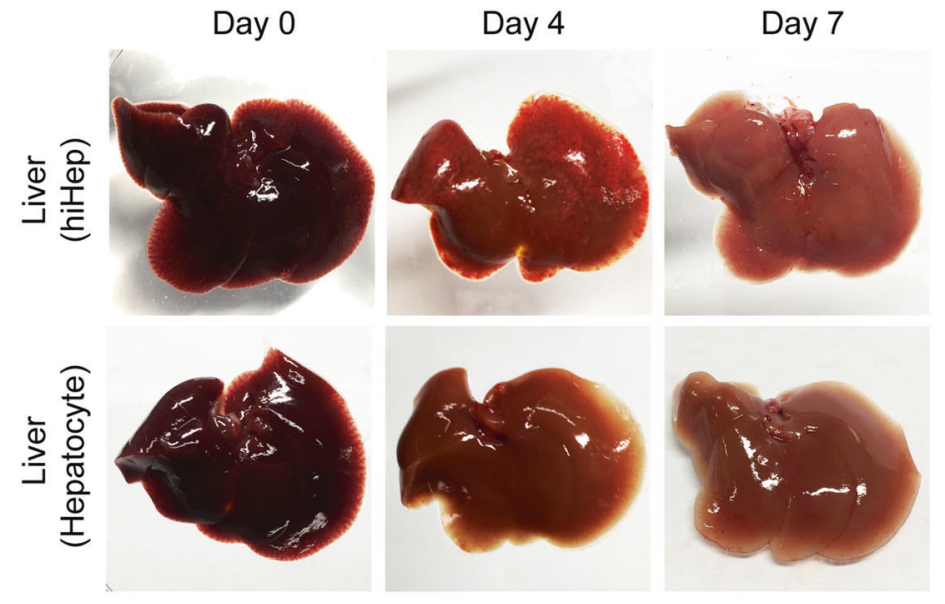

d

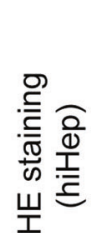

Day 0

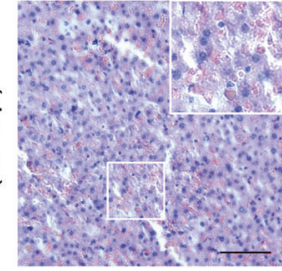

Day 4

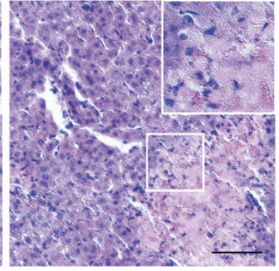

Day 7

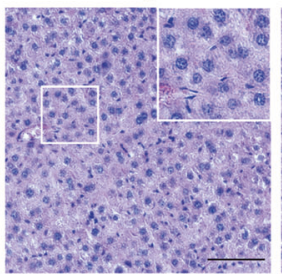

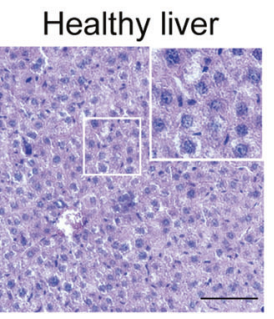

e
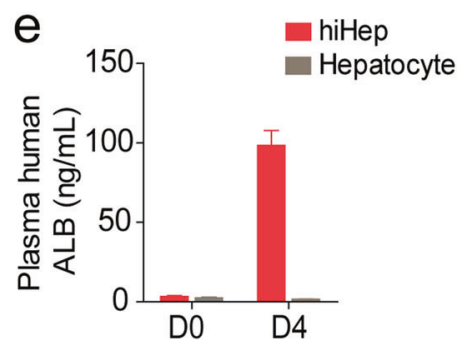

Fig. 5 Rescue of acute liver failure by Foxa3-T-6C-hiHeps. a Kaplan-Meier survival curves of SCID mice injected with ConA and then transplanted with hUCs $(n=10)$, Foxa3-T-6C-hiHeps $(n=12)$, or primary mouse hepatocytes $(n=9)$. ${ }^{*} P<0.05$, ${ }^{* *} P<0.005$, log-rank test. b Plasma levels of ALT, AST, and total bilirubin in ConA-induced acute liver failure mice before (day 0) and after (day 4 and day 7) transplantation of Foxa3-T-6C-hiHeps or primary mouse hepatocytes. c Representative image of the whole liver in ConA-treated mice before (day 0 ) and after (day 4 and day 7) transplantation of Foxa3-T-6C-hiHeps or hepatocytes. d Hematoxylin and eosin staining of liver sections of ConA-treated mice before (day 0) and after (day 4 and day 7) transplantation of Foxa3-T-6C-hiHeps. Note: ConA-induced hemorrhage in the liver at day 0, residual liver damage in the mice injected with Foxa3-T-6C-hiHeps at day 4, and complete liver recovery at day 7. Scale bars represent $100 \mu \mathrm{m}$. e Human albumin levels were measured in the serum of recipient mice after transplantation of Foxa3-T-6C-hiHeps or primary mouse hepatocytes at day 4

transplanted into the mice via tail-vein injection. All 10 ConAinduced liver failure mice transplanted with hUCs died within $6 \mathrm{~h}$. In contrast, 4 of 9 mice receiving primary mouse hepatocytes and 3 of 12 mice receiving Foxa3-T-6C-hiHeps survived to the end of the experiment (7 days, Fig. 5a). Moreover, serum levels of total ALT, AST, and total bilirubin gradually decreased in both Foxa3-T$6 \mathrm{C}$-hiHeps- and mouse hepatocyte-recipient mice (Fig. 5b). Mouse livers showed severe signs of hemorrhage and inflammation after ConA injection (day 0, Fig. 5c, d). After hepatocyte or Foxa3-T-6ChiHeps transplantation, recovery of liver tissue was observed at day 4 and day 7 (Fig. 5c, d). To confirm that hiHeps did function in vivo as hepatocytes after transplantation, human albumin was measured. As demonstrated in Fig. 5e, 4 days after tail-vein injection of Foxa3-T-6C-hiHeps, human albumin was detectable in the serum of recipient mice. In conclusion, these data further demonstrated that Foxa3-T-6C-hiHeps are functional in vivo and show therapeutic effects in ALF mice.

\section{DISCUSSION}

In this study, we demonstrated that Foxa3, Hnf1a, or Hnf4a alone was sufficient to generate functional hiHeps from hUCs in the presence of the chemical cocktail CRVPTD. Although previous investigations have shown that functional hiHeps can be induced from human fibroblasts via ectopic TF expression [11, 15], identifying chemical cocktails that can substitute for TFs in hepatic transdifferentiation of human cells remains a worthy goal.

Small molecules have been reported to play important roles in cell fate determination, including stem cell self-renewal, differentiation, and somatic cell reprogramming $[16,29,30]$. The chemical cocktail CRVPTD was modified from the original cocktail CRFVPTD (F, forskolin), which was first reported to generate chemically induced pluripotent stem cells (CiPSCs) from MEFs without any TFs [29]. We found the chemical cocktail CRFVPTD can also lead to the generation of mouse chemically induced cardiomyocytes (CiCMs) under different culture conditions [31]. Our previous 
work also demonstrated that forskolin should be removed from the cocktail when inducing iHeps from mouse fibroblasts with one $\mathrm{TF}$, Foxa1, 2, or 3 [16] because it may reduce the transcriptional activity of endogenous Hnf4a through phosphorylation [32] by activating the CAMP pathway. Here, we show that the same cocktail CRVPTD (6C) also led to the hepatic transdifferentiation of hUCs with one TF, Foxa3, Hnf1a, or Hnf4a.

Considering many of the compounds within the $6 C$ cocktail overlap with previously reported cocktails used to induce CiPSCs [29] and CiCMs [31], in addition to the fact that we observed the appearance of various morphological changes at the early stage of CiCM induction [31], we previously hypothesized that this chemical combination might induce the generation of a mixedintermediate cell population. When favorable culture conditions are provided, these intermediate cells can be induced to become pluripotent or to differentiate into various functional cells [31]. Although the exact mechanism underlying a particular conversion between two types of cells remains unclear, we hypothesize that a typical cocktail used for full chemical reprogramming should contains three parts: epigenetic modulators, compounds that suppress the characteristics of the starting cells, and compounds that induce the characteristics of the designated cells [33]. However, we failed to generate iHeps with only chemicals in both mouse [16] and human systems; one TF had to remain to obtain hepatocyte-like cells. Thus, we still appear to lack the perfect compounds or culture conditions that can push the intermediate cell population to become mature hepatocytes, and the remaining one TF, Foxa3, Hnfla, or Hnf4a serves as this maturation or fate determination factor.

In the original hiHeps study, the presence of all three TFs (Foxa3, $H n f 1 a$, and Hnf4a) was necessary to induce hepatic transdifferentiation of human fibroblasts [15]. Removal of any factor inhibited the hepatic transdifferentiation. However, any two TFs were able to be replaced with chemical cocktail CRVPTD in our study, as long as one of the TFs was present, indicating potentially overlapping roles of these TFs in hepatocyte fate determination. Forkhead box A proteins (Foxa1/2/3), also known as Hnf3, function as pioneer factors to open compacted chromatin and seed the transcriptional and epigenetic complexes of liver-specific genes [34]. Foxa3 is the most highly expressed of the FoxA family in the adult liver [35] and works as a transcriptional activator for liverspecific transcripts such as albumin and transthyretin. Foxa3 also plays an important role in the maintenance of glucose homeostasis [36]. Hnf1a and Hnf4a are important for hepatocyte differentiation. Hnf1a binds to many hepatic promoters and regulates hepatic function, including carbohydrate synthesis and storage, lipid metabolism, detoxification, and serum protein synthesis [37]. Hnf1a also regulates its own expression in hepatocytes [38]. During liver development, Hnf4a serves as a master regulator of hepatocyte differentiation and the maintenance of liver functions [39]. Hnf4a binds to a large number of promoters of actively transcribed genes in the liver and occupies most of the promoters bound by Hnf1a $[37,40]$. Hnf4a is essential for maintaining triglyceride and cholesterol homeostasis [41]. Hnf4a deficiency also causes a defect in mature hepatocyte gene expression.

In our previous study, mouse iHeps generated with one TF and the chemical cocktail were successfully expanded for $>30$ passages in vitro [16]. However, the hUC-derived hiHeps displayed proliferation arrest. Proliferation arrest is a major barrier for the application of human terminally differentiated cells generated by direct reprogramming $[11,15,42]$. The large $T$ antigen had to be introduced to obtain T-hiHeps that can be expanded for at least 20 passages. Introduction of large $T$ antigen does not affect the functions of these hiHeps. The expandable T-hiHeps provided us the opportunity to demonstrate the therapeutic effects of these cells in an ALF animal model. Although the expression of large $T$ in hiHeps is not a concern for the application in bioartificial liver supporting devices [43] or in vitro disease modeling, other measures to expand hiHeps for cell therapies are still needed. Our hiHeps generated using one TF and a chemical cocktail are functional to a certain extent, but compared with primary hepatocytes, there are still some gaps, such as the expression levels of hepatic-feature genes, drug metabolism ability, and in vivo function. More efforts will be needed to improve the functions of these hiHeps.

In conclusion, we generated functional and expandable human hepatocytes using a single TF plus a chemical cocktail and are one step closer to TF-free hiHeps. Future mechanistic studies and further drug screening will help provide insights into the roles of chemicals in human hepatic transdifferentiation and eventually lead to full chemical induction.

\section{ACKNOWLEDGEMENTS}

This work was supported by grants from the Ministry of Science and Technology of China (2015CB964503, 2017YFA0104002), the Chinese Academy of Sciences (XDA01040301), and the National Natural Science Foundation of China (81425024, 31371511, 81472862, and 31501189).

\section{AUTHOR CONTRIBUTIONS}

WT and RG conducted most of the experiments, analyzed the results, and wrote the paper; SJS and CZJ analyzed the microarray data; YZ, YTL, MMJ, XC provided assistance in different experiments; XX conceived the idea for the project, analyzed the results, and wrote the paper. All authors reviewed the results and approved the final version of the manuscript.

\section{ADDITIONAL INFORMATION}

The online version of this article (https://doi.org/10.1038/s41401-018-0170-z) contains supplementary material, which is available to authorized users.

Competing interests: The authors declare no competing interests.

\section{REFERENCES}

1. Touboul T, Hannan NR, Corbineau S, Martinez A, Martinet C, Branchereau S, et al. Generation of functional hepatocytes from human embryonic stem cells under chemically defined conditions that recapitulate liver development. Hepatology. 2010:51:1754-65.

2. Soltys KA, Soto-Gutierrez A, Nagaya M, Baskin KM, Deutsch M, Ito R, et al. Barriers to the successful treatment of liver disease by hepatocyte transplantation. J Hepatol. 2010;53:769-74.

3. Merion RM. When is a patient too well and when is a patient too sick for a liver transplant? Liver transplantation: official publication of the American Association for the Study of Liver Diseases and the International Liver Transplantation. Society. 2004;10:S69-73.

4. Dolgikh MS. [The clinical experience with hepatocyte transplantation for the treatment of hepatic insufficiency]. Klin Med (Mosk). 2012;90:18-22.

5. De Vree JM, Ottenhoff R, Bosma PJ, Smith AJ, Aten J, Oude Elferink RP. Correction of liver disease by hepatocyte transplantation in a mouse model of progressive familial intrahepatic cholestasis. Gastroenterology. 2000;119:1720-30.

6. Kobayashi N, Fujiwara T, Westerman KA, Inoue Y, Sakaguchi M, Noguchi $H$, et al. Prevention of acute liver failure in rats with reversibly immortalized human hepatocytes. Science. 2000;287:1258-62.

7. Kocken JM, Borel Rinkes IH, Bijma AM, de Roos WK, Bouwman E, Terpstra OT, et al. Correction of an inborn error of metabolism by intraportal hepatocyte transplantation in a dog model. Transplantation. 1996;62:358-64.

8. Cai J, Zhao Y, Liu Y, Ye F, Song Z, Qin H, et al. Directed differentiation of human embryonic stem cells into functional hepatic cells. Hepatology. 2007;45:1229-39.

9. Rambhatla L, Chiu CP, Kundu P, Peng Y, Carpenter MK. Generation of hepatocytelike cells from human embryonic stem cells. Cell Transplant. 2003;12:1-11.

10. Takebe $T$, Sekine $K$, Enomura $M$, Koike $H$, Kimura M, Ogaeri $T$, et al. Vascularized and functional human liver from an iPSC-derived organ bud transplant. Nature. 2013;499:481-4.

11. Du Y, Wang J, Jia J, Song N, Xiang C, Xu J, et al. Human hepatocytes with drug metabolic function induced from fibroblasts by lineage reprogramming. Cell Stem Cell. 2014;14:394-403.

12. Shiota G, Yasui T. Progress in stem cell biology in regenerative medicine for liver disease. Hepatol Res. 2012;42:15-21. 


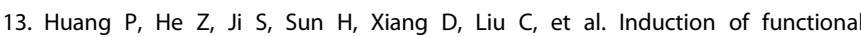
hepatocyte-like cells from mouse fibroblasts by defined factors. Nature. 2011;475:386-9.

14. Sekiya S, Suzuki A. Direct conversion of mouse fibroblasts to hepatocyte-like cells by defined factors. Nature. 2011;475:390-3.

15. Huang P, Zhang L, Gao Y, He Z, Yao D, Wu Z, et al. Direct reprogramming of human fibroblasts to functional and expandable hepatocytes. Cell Stem Cell. 2014;14:370-84.

16. Guo R, Tang W, Yuan Q, Hui L, Wang X, Xie X. Chemical cocktails enable hepatic reprogramming of mouse fibroblasts with a single transcription factor. Stem Cell Rep. 2017;9:499-512.

17. Simeonov KP, Uppal H. Direct reprogramming of human fibroblasts to hepatocyte-like cells by synthetic modified mRNAs. PLoS ONE. 2014;9:e100134.

18. Shi XL, Gao Y, Yan Y, Ma H, Sun L, Huang P, et al. Improved survival of porcine acute liver failure by a bioartificial liver device implanted with induced human functional hepatocytes. Cell Res. 2016;26:206-16.

19. Lim KT, Lee SC, Gao YM, Kim KP, Song GQ, An SY, et al. Small molecules facilitate single factor-mediated hepatic reprogramming. Cell Rep. 2016;15:814-29.

20. Wang L, Wang L, Huang W, Su H, Xue Y, Su Z, et al. Generation of integration-free neural progenitor cells from cells in human urine. Nat Methods. 2013;10:84-9.

21. Zhou T, Benda C, Dunzinger S, Huang Y, Ho JC, Yang J, et al. Generation of human induced pluripotent stem cells from urine samples. Nat Protoc. 2012;7:2080-9.

22. Zhou T, Benda C, Dunzinger S, Huang YH, Ho JC, Yang JY, et al. Generation of human induced pluripotent stem cells from urine samples. Nat Protoc. 2012;7:2080-9.

23. Wang LH, Wang LL, Huang WH, Su HX, Xue YT, Su ZH, et al. Generation of integration-free neural progenitor cells from cells in human urine. Nat Methods. 2013;10:84-U124.

24. Jia C. Advances in the regulation of liver regeneration. Expert Rev Gastroenterol Hepatol. 2011;5:105-21.

25. Stravitz RT, Kramer DJ. Management of acute liver failure. Nature reviews. Gastroenterol Hepatol. 2009;6:542-53.

26. Ostapowicz G, Lee WM. Acute hepatic failure: a Western perspective. J Gastroenterol Hepatol. 2000;15:480-8.

27. Torisu T, Nakaya M, Watanabe S, Hashimoto $M$, Yoshida $H$, Chinen $T$, et al. Suppressor of cytokine signaling 1 protects mice against concanavalin A-induced hepatitis by inhibiting apoptosis. Hepatology. 2008:47:1644-54.

28. Sun L, Fan X, Zhang L, Shi G, Aili M, Lu X, et al. Bone mesenchymal stem cell transplantation via four routes for the treatment of acute liver failure in rats. Int $J$ Mol Med. 2014;34:987-96.
29. Hou PP, Li YQ, Zhang X, Liu C, Guan JY, Li HG, et al. Pluripotent stem cells induced from mouse somatic cells by small-molecule compounds. Science. 2013;341:651-4.

30. Long $\mathrm{Y}$, Wang $\mathrm{M}, \mathrm{Gu} \mathrm{HF}$, Xie X. Bromodeoxyuridine promotes full-chemical induction of mouse pluripotent stem cells. Cell Res. 2015;25:1171-4.

31. Fu YB, Huang CW, Xu XX, Gu HF, Ye YQ, Jiang CZ, et al. Direct reprogramming of mouse fibroblasts into cardiomyocytes with chemical cocktails. Cell Res. 2015;25:1013-24.

32. Viollet B, Kahn A, Raymondjean M. Protein kinase A-dependent phosphorylation modulates DNA-binding activity of hepatocyte nuclear factor 4. Mol Cell Biol. 1997;17:4208-19.

33. Xie $X, F u$ Y, Liu J. Chemical reprogramming and transdifferentiation. Curr Opin Genet Dev. 2017;46:104-13.

34. Gordillo M, Evans T, Gouon-Evans V. Orchestrating liver development. Development. 2015;142:2094-108.

35. Kaestner $\mathrm{KH}$, Hiemisch $\mathrm{H}$, Luckow B, Schutz G. The HNF-3 gene family of transcription factors in mice: gene structure, cDNA sequence, and mRNA distribution. Genomics. 1994;20:377-85.

36. Shen W, Scearce LM, Brestelli JE, Sund NJ, Kaestner KH. Foxa3 (hepatocyte nuclear factor 3gamma) is required for the regulation of hepatic GLUT2 expression and the maintenance of glucose homeostasis during a prolonged fast. J Biol Chem. 2001;276:42812-7.

37. Odom DT, Zizlsperger N, Gordon DB, Bell GW, Rinaldi NJ, Murray HL, et al. Control of pancreas and liver gene expression by HNF transcription factors. Science. 2004;303:1378-81.

38. Miura N, Tanaka K. Analysis of the rat hepatocyte nuclear factor (HNF) 1 gene promoter: synergistic activation by HNF4 and HNF1 proteins. Nucleic Acids Res. 1993;21:3731-6.

39. Lu H. Crosstalk of HNF4alpha with extracellular and intracellular signaling pathways in the regulation of hepatic metabolism of drugs and lipids. Acta Pharm Sin B. 2016:6:393-408.

40. Lau HH, Ng NHJ, Loo LSW, Jasmen JB, Teo AKK. The molecular functions of hepatocyte nuclear factors - in and beyond the liver. J Hepatol. 2018;68: 1033-48.

41. Hayhurst GP, Lee YH, Lambert G, Ward JM, Gonzalez FJ. Hepatocyte nuclear factor 4alpha (nuclear receptor 2A1) is essential for maintenance of hepatic gene expression and lipid homeostasis. Mol Cell Biol. 2001;21:1393-403.

42. Graf T, Enver T. Forcing cells to change lineages. Nature. 2009;462:587-94.

43. Carpentier B, Gautier A, Legallais C. Artificial and bioartificial liver devices: present and future. Gut. 2009;58:1690-702. 